Document downloaded from:

http://hdl.handle.net/10251/178661

This paper must be cited as:

Sánchez-Escuderos, D.; Herranz Herruzo, JI.; Ferrando-Rocher, M.; Valero-Nogueira, A. (2020). Mechanical phase shifter in gap-waveguide technology. IEEE. 1-5. https://doi.org/10.23919/EuCAP48036.2020.9135719

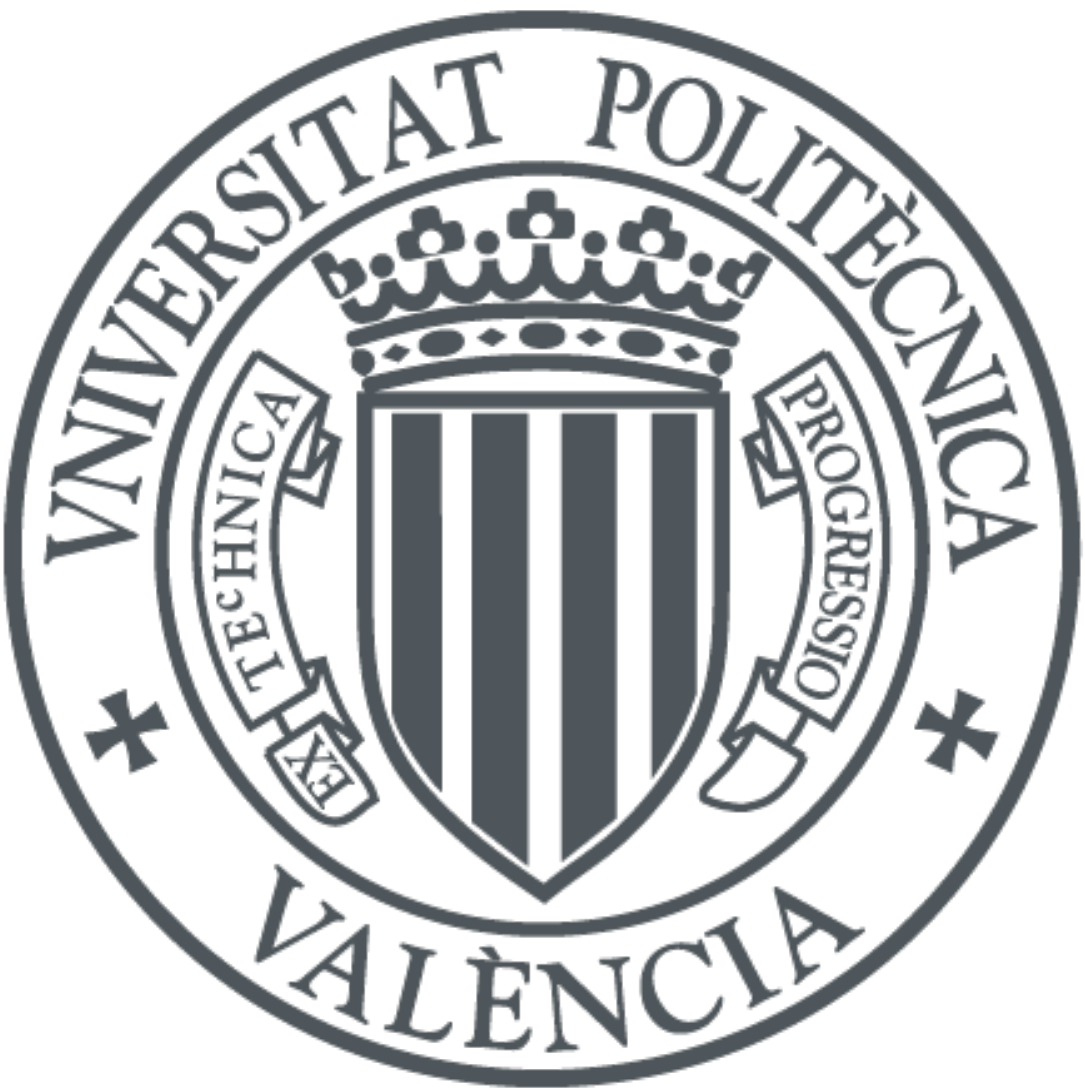

The final publication is available at

https://doi.org/10.23919/EuCAP48036.2020.9135719

Copyright IEEE

Additional Information 


\title{
Mechanical phase shifter in gap-waveguide technology
}

\author{
Daniel Sánchez-Escuderos*, Jose I. Herranz-Herruzo*, Miguel Ferrando-Rocher*, Alejandro Valero-Nogueira* \\ *Instituto de Telecomunicaciones y Aplicaciones Multimedia (iTEAM), Universitat Politècnica de València. \\ c/ Cami de Vera s/n 46022 Valencia (Spain). dasanes1@iteam.upv.es
}

\begin{abstract}
This contribution presents a low-loss mechanical phase shifter in gap-waveguide technology. The phase shifter is aimed at ground terminals for Ka-band satellite on-the-move applications. The use of the gap-waveguide technology allows to divide the device into two main blocks distributed in two levels: a lower-rotatable block, in charge of the power distribution and the phase shifting; and an upper-fixed block with the output waveguides. In this paper, the lower and upper blocks are designed using Groove-gap waveguides (GGW), and Ridge-gap waveguides, respectively, connected to each other by coupling slots. Results show a good performance within the operating frequency band in terms of the phase shift between consecutive output ports (suitable for array feeding), and the return loss level at the input port.
\end{abstract}

Index Terms-Gap-waveguide technology, Phase shifter, satellite communications, ground terminals.

\section{INTRODUCTION}

Satellite-on-the-move (SOTM) communication systems provide high-speed Internet connection in mobile environments such as trains, aircrafts or emergency vehicles [1]. In order to attend the high-data rate demands of current and future users, SOTM systems are currently being implemented in the $\mathrm{K}$ (around $20 \mathrm{GHz}$ ) and $\mathrm{Ka}$ (around $30 \mathrm{GHz}$ ) bands for the downlink and uplink, respectively [2].

SOTM systems are embarked in geostationary satellites to provide a multiple-beam coverage over large areas [3]. Ground terminals for users in movement must perform a dynamic tracking of the satellite in order to point the maximum gain of the ground antenna to the satellite.

Systems currently deployed make use of several engines to rotate and tilt the antenna to steer the main beam towards the satellite [4]. Recently, a low-cost low-profile solution has been proposed [5] in this area. The low profile is obtained by substituting traditional reflectors by slot-array antennas. However, the use of several mechanical engines is still required in this solution. These engines provide a good performance, but they need to move heavy structures, which often may compromise its accuracy, reliability and durability.

Alternatively, mechanical engines can be substituted by electronic phase shifters. These devices can be used to change the phase profile of the antenna elements and, in turn, the pointing of the main beam [6]. The major drawback of the electronic phase shifters is their high losses [7], which may heavily degrade the system performance.

This paper proposes a new all-metallic phase shifter as an intermediate solution between the aforementioned structures,

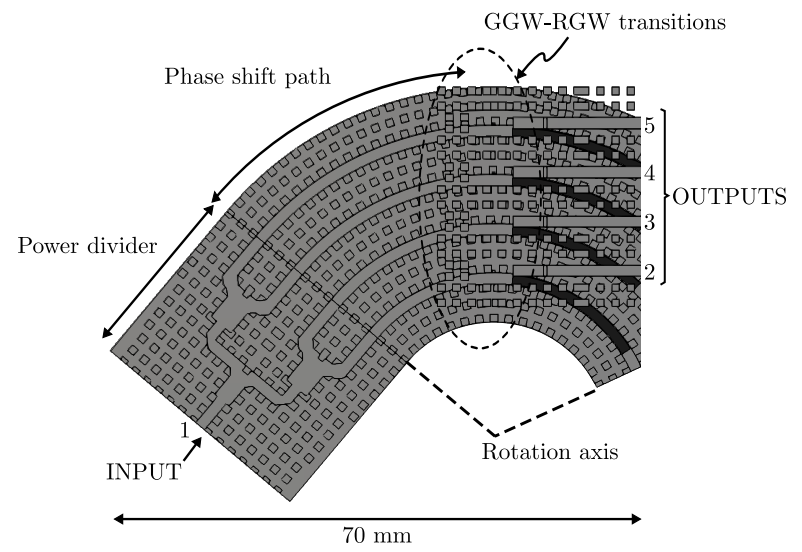

Fig. 1. Upper view of the complete phase shifter, without metallic covers.

in which the beam pointing is obtained by changing the phase shift between consecutive elements of the antenna. In order to adjust dynamically this phase shift, the length of the feeding waveguides is changed by rotating mechanically the lower block of the system. Since only the lower part of the antenna is moved mechanically, the radiating elements are fixed, thereby keeping the low profile feature of the antenna for any pointing angle. Furthermore, the use of all-metallic phase shifters, instead of electric phase shifters, reduces considerably the losses of the system. In [8], a similar solution is described, but the required waveguide bends leads to a hardly-implementable solution in practice.

The phase shifter is designed in gap-waveguide technology [9]. This technology has already been used to design a mechanical phase shifter formed by several layers [10]. The main advantage of our proposal is that we only need two levels with a bed of nails, which facilitates the fabrication of the device. In particular, the lower block, in charge of the phase shifting, is designed with Groove gap waveguides (GGW), and the upper block, which eventually will be connected to the radiating elements, with Ridge gap waveguides (RGW). A small gap is left between the two blocks to allow the rotation of the lower GGW block with respect to the upper RGW block. This gap does not produce leakage because propagation of waves is prohibited between the lower bed of nails and the upper metallic plane [9]. 


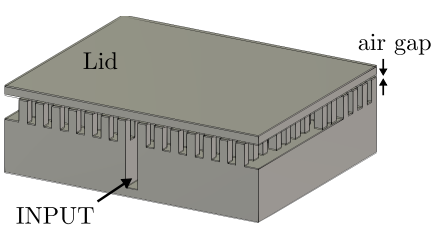

(a)

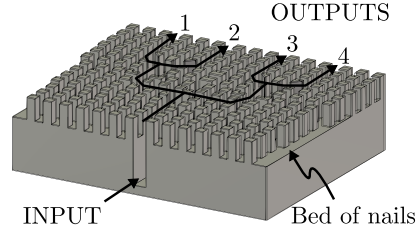

(b)
Fig. 2. 3D view of the 1:4 power divider in GGW: (a) with cover, and (b) without cover.

\section{DESCRIPTION OF THE PHASE SHIFTER}

The proposed phase shifter is aimed at distributing the input signal among the $N$ output ports with a certain phase shift. The outputs will, eventually, be connected to different antenna elements so that the phase shift will steer the beam to obtain the desired pointing. Fig. 1 shows an upper view of the phase shifter proposed. As we can see, the structure is formed by three main blocks: a power divider (input port), phaseshifting waveguides, and GGW-RGW transitions (output ports are RGW-type).

The three blocks in which the phase shifter is decomposed are distributed in two levels: an upper fixed level, and a lower level capable of performing a rotation movement. The upper level is only formed by the output RGW waveguides [9] and the transition from the lower level to the upper level. The lower blocks are the power divider and the phase-shifting GGW around the rotation axis [11]. The rotation around this axis varies the GGW electrical length between the output ports of the power divider and the transition to the upper level, thereby causing a variation of the phase shift between consecutive output RGWs.

In order to have an upper fixed block, and a lower rotatable block, the use of the gap-waveguide technology becomes key. This technology is based on the high-impedance condition produced on the upper plane of a bed of nails [9]. If a metallic plane is placed above this bed of nails with a separation smaller than $\lambda / 4$, propagation of waves in the gap between the bed of nails and the metallic plane is forbidden. Hence, the field is confined within the groove of the GGW or in the gap between the ridge of the RGW and the upper metallic lid. This behavior allows to leave a small gap between the lower GGW devices and the upper RGW outputs without leakage.

\section{A. Power divider}

The power divider is in charge of splitting the input signal into $N$ output ports with the same phase, being $N$ the number of output RGW. Figs. 2 and 3 show a power divider for $N=4$ in GGW. As we can see, a tree topology is chosen to maximize the bandwidth, and guarantee the same phase and amplitude in all output ports.

GGWs are created by means of grooves in a bed of nails (labeled as GGW in Fig. 3). As indicated above, the use of a bed of nails allows us to leave a small gap between the bed of nails and the back of the upper block (see Fig. 2a) without any sort of leakage [11]. In particular, a $200 \mu \mathrm{m}$ gap has been

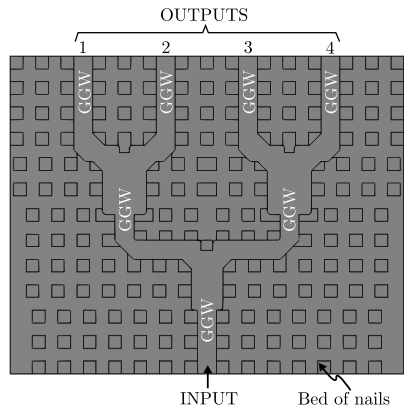

Fig. 3. Upper view of the 1:4 power divider in GGW without cover.

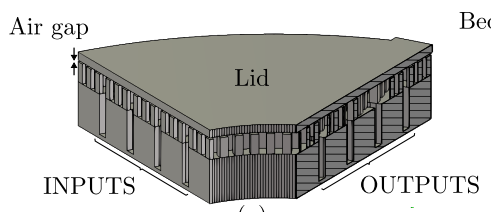

(a)

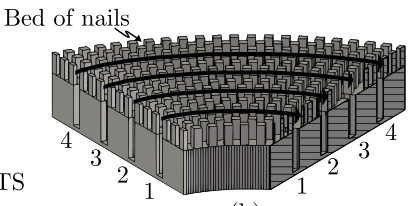

(b)
Fig. 4. 3D view of the phase-shifting GGWs: (a) with metallic cover, and (b) without metallic cover.

considered in this design, which is smaller than $\lambda / 4$ at the operating frequency band (29.5-30.5 GHz).

\section{B. Phase-shifting waveguides.}

Phase-shifting GGWs implement the desired phase shift to the different channels. To do so, $N$ GGW are connected to the output ports of the 1:N power divider. These concentric GGWs are deployed following a circular path around a rotation axis. Therefore, all input ports are placed on the same plane, and all the output ports are on another plane with a rotation angle $\alpha$ with respect to the first plane. The phase shift between consecutive output ports, which depends on the length of the GGW paths, i.e., on $\alpha$, will be identical as long as the difference of curvature radius between consecutive output ports $R_{i}$ is identical.

Figs. 4 y 5 show an example of phase shifting GGW for $N=4$. The phase shift between the waveguide $i$ and $j\left(\phi_{i, j}\right)$ can be calculated from the propagation constant of the GGW $\left(\beta_{G G W}\right)$ and the difference of GGW path lengths $\left(L_{i, j}\right)$ as:

$$
\phi_{i, j}=\beta_{G G W} L_{i, j}
$$

where $L_{i, j}$ can be calculated as:

$$
L_{i, j}=\left(R_{j}-R_{i}\right) \alpha
$$

being $R_{i}$ the curvature radius of each GGW (see Fig. 5).

\section{Output: $G G W-R G W$ transition}

The output ports of the phase shifter are located at the end of the phase-shifting GGW. These ports are placed on an upperfixed level, so that the floor of the output ports is the lid of the lower power divider and the phase-shifting GGWs. As commented above, the gap between these two blocks allows 


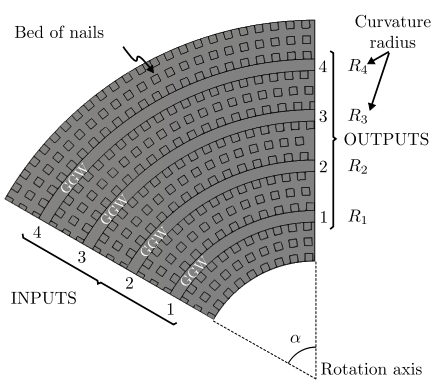

Fig. 5. Upper view of the phase-shifting GGWs without metallic cover.

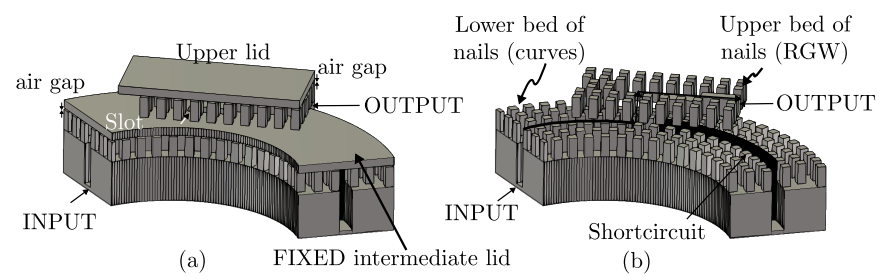

Fig. 6. 3D view of the two-levels GGW-RGW transition: (a) with metallic cover and (b) without metallic cover.

to rotate the lower devices, keeping the upper output ports fixed.

The output ports are RGWs, which will be connected to the antenna elements in a later stage. We need to design a transition from the lower phase-shifting GGW to the upper RGW. Figs. 6 and 7 show a sketch of the transition proposed in this paper. As we can see, the transition is formed by three parts.

The first part is a metallic protrusion attached to the intermediate metallic plane acting as RGW floor and GGW lid. The protrusion has the same circular shape as the lower GGW, and is inserted inside the groove of the GGW. This element produces a shortcircuit (as labeled in Fig. 6b) at the end of the phase-shifting GGW to reflect all the energy. The attachment of the shortcircuit to the intermediate metallic plane, and its circular shape, allows to keep this element always at the same point with respect to the upper outputs, despite the rotation of the lower block. Thereby, this movement produces an effective variation of the length of the phase-shifting GGW, from the output ports of the power divider to the shortcircuit.

The second part is a slot in the intermediate metallic plane. This slot is placed at an optimal distance from the shortcircuit to have a maximum electric field. The aim of the slot is to couple the energy from the lower GGW level to the upper RGW level. On the edge of the slot is located the third part of the transition, a coaxial nail. This nail is the center of a coaxial cavity in gap waveguide technology on the upper face of the intermediate metallic plane (see Fig. 7) [12]. The cavity is used to couple the field transmitted by the slot to the output RGW.

\section{PHASE SHITER PERFORMANCE}

The complete phase shifter is formed by the three elements described in previous section. Fig. 8 shows a 3D view of the

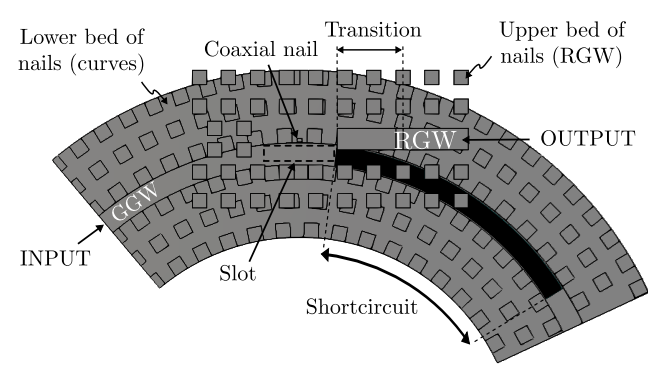

Fig. 7. Upper view of the two-levels GGW-RGW transition.

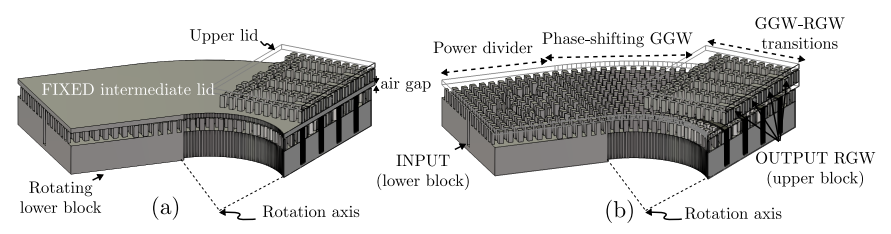

Fig. 8. 3D view of the complete phase shifter: (a) without upper RGW cover, and (b) without upper RGW and intermediate cover.

phase shifter for $N=4$. In this figure, the upper cover of the RGW (Fig. 8a), and the intermediate metallic plane between the GGW and the RGW (Fig. 8b) have not been included for a better visualization of the inner parts of the phase shifter. Fig. 1 shows an upper view of the complete structure.

In general, the operation mode of the phase shifter starts with the feeding of the input port of the power divider (see Fig. 8b). This signal is split into $N$ output signals that feed the phase-shifting GGW. Since the length of the $N$ concentric GGW is different, the phase at the end of the GGW paths is different. At this point, the slot at the end of the GGW couples the field to the upper level. This field excites a coaxial cavity on the upper block which, in turn, excites the output RGW.

The phase shifter has been designed in parts (power divider, phase-shifting GGW and RGW-GGW transition), and, later, assembled in CST Microwave Studio. Fig. 9 shows the $\mathrm{S}_{11}$ parameter at the input port of the complete phase shifter for several rotation angles $\alpha$. As we can see, matching is below $-15 \mathrm{~dB}$ from $29.5 \mathrm{GHz}$ to $30.5 \mathrm{GHz}$.

The insertion loss in all output ports is flat along the whole operation frequency band, around $6 \mathrm{~dB}$ due to the 1:4 power division. Fig. 10 shows the $S_{31}$ parameter for several rotation angles (see the ports labels in Fig. 1).

In order to assess the phase shift produced by the device proposed in this paper, we need to calculate the difference between the phase of the $S_{n 1}$ parameters in consecutive output ports. Since the increment of curvature radius between all consecutive phase-shifting GGW is constant, the phase difference between consecutive output ports is the same for any pair of ports. Thereby, we only need to study one pair of consecutive ports. In particular, Fig. 11 shows the phase difference between output ports 3 and 4 for several rotation angles, $\alpha$, and compares these simulated values with the expected phase difference according to (1).

As we can see in Fig. 11, the phase difference shows 


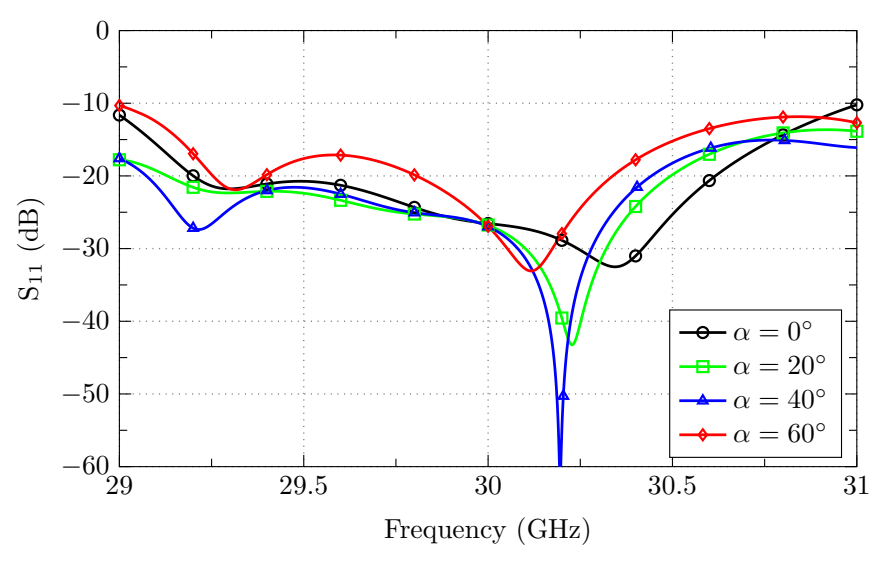

Fig. 9. $\mathrm{S}_{11}$ parameter of the complete phase shifter for several rotation angles $(\alpha)$

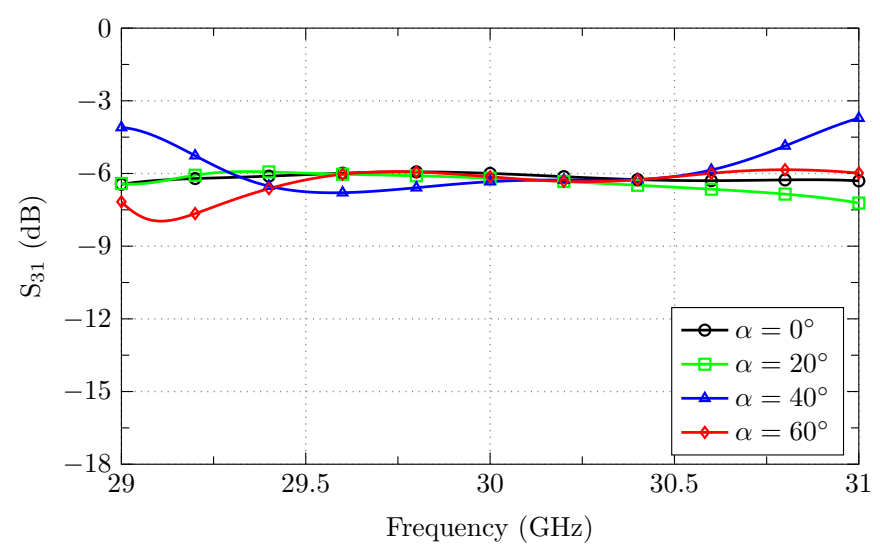

Fig. 10. $\mathrm{S}_{31}$ parameter of the complete phase shifter for several rotation angles $(\alpha)$.

small oscillations around the expected values. By increasing the rotation angle, the phase difference is increased since the difference between path lengths is larger.

The phase shift is also illustrated in Fig. 12, where it is represented the instantaneous electric field on two levels of the complete phase shifter. Fig. 12a represents the electric field in the power divider and in the phase-shifting GGWs, and Fig. 12b shows the electric field in the RGW above. In both cases, we can see how the field has the same phase in all the GGWs at the output of the power divider. However, at the end of the phase-shifting GGW and in the output RGW, there is a small phase shift between the different waveguides.

\section{CONCLUSIONS}

This work presents an all-metallic phase shifter capable of controlling the phase difference between consecutive elements of an array antenna. The phase shifter has been implemented in gap waveguide technology to split the structure into two parts: a lower-rotating block, which changes the length of the feeding lines, and an upper-fixed block, where the output RGW are located. These RGW will be later connected to the elements of the array antenna.

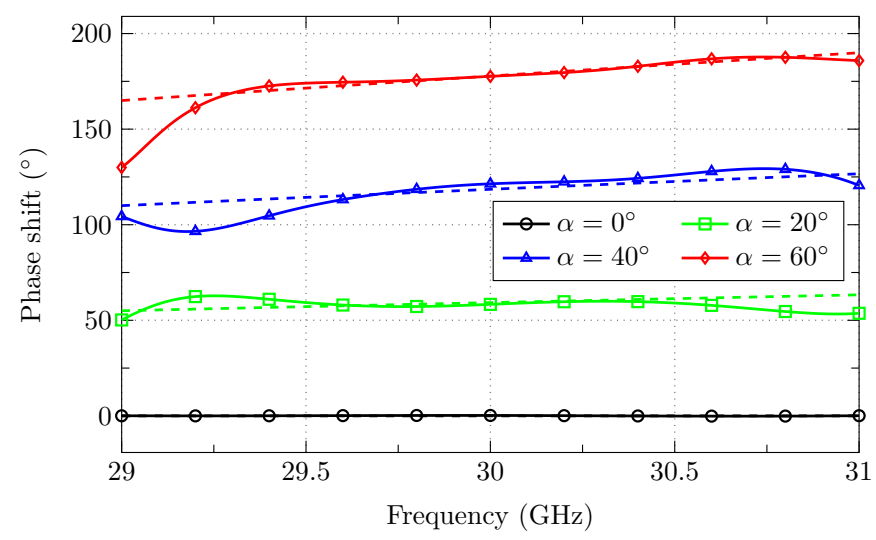

Fig. 11. Phase difference between output ports 3 and 4 for several rotation angles $\alpha$. Solid lines correspond to the simulation of the complete phase shifter, and dashed lines correspond to the expected phase difference according to the theoretical formulas.

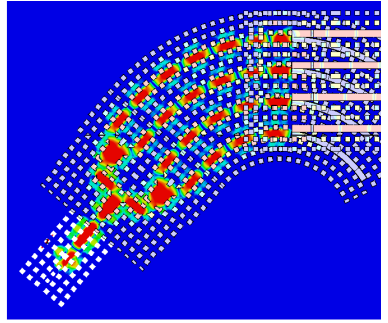

(a)

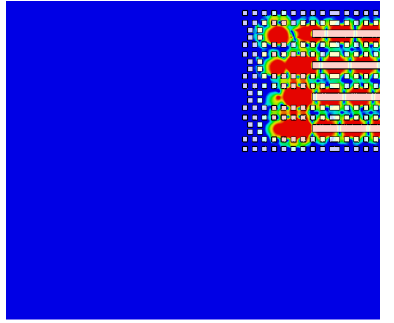

(b)
Fig. 12. Electric field at $30 \mathrm{GHz}$ for a rotation angle of $\alpha=50^{\circ}$ on two levels of the phase shifter: (a) on the lower power divider and phase-shifting GGW, and (b) on the upper RGW.

The advantage of the phase shifter presented in this paper is that it only requires the mechanical movement of part of the structure, leaving the output ports and the antenna fixed. Thereby, the positioning system needs to do less complicated mechanical movements, which will reduce the cost of the tracking systems.

\section{ACKNOWLEDGMENT}

This work has been supported by the Spanish Ministry of Science, Innovation and Universities (Ministerio de Ciencia, Innovacion y Universidades) under project TEC2016-79700C2-1-R.

\section{REFERENCES}

[1] R. L. Forti et al., "Mobile communications: High-speed train antennas from $\mathrm{Ku}$ to Ka," in 5th European Conference on Antennas and Propagation, 2011, pp. 2354-2357.

[2] H. Fenech, S. Amos, A. Tomatis, and V. Soumpholphakdy, "High throughput satellite systems: An analytical approach," IEEE Transactions on Aerospace and Electronic Systems, vol. 51, no. 1, pp. 192-202, 2015.

[3] S. K. Rao, "Advanced antenna technologies for satellite communications payloads," IEEE Trans. Antennas Propag., vol. 63, no. 4, pp. 1205-1217, 2015.

[4] "EM solutions," urlhttps://www.emsolutions.com.au/, 2019 
[5] J. I. Herranz-Herruzo, A. Valero-Nogueira, M. Ferrando-Rocher, B. Bernardo, A. Vila, and R. Lenormand, "Low-cost Ka-band switchable RHCP/LHCP antenna array for mobile SATCOM terminal," IEEE Transactions on Antennas and Propagation, vol. 66, no. 5, pp. 26612666, 2018.

[6] A. Malczewski, S. Eshelman, B. Pillans, J. Ehmke, and C. Goldsmith, "X-band RF MEMS phase shifters for phased array applications," IEEE Microwave and Guided Wave Letters, vol. 9, no. 12, pp. 517-519, 1999.

[7] S. Alessandro et al., "Analog beamforming network for Ka band satellite on the move terminal with phase shifting technique based on I/Q mixer," in 2015 European Radar Conference, 2015, pp. 445-448.

[8] X. Fang, Y. Huang, and G. Wen, "A low-cost mechanical beam scanning waveguide slot antenna array," in 2017 IEEE International Symposium on Antennas and Propagation \& USNC/URSI National Radio Science Meeting. IEEE, 2017, pp. 2603-2604.

[9] P.-S. Kildal, E. Alfonso, A. Valero-Nogueira, and E. Rajo-Iglesias, "Local metamaterial-based waveguides in gaps between parallel metal plates," IEEE Antennas and Wireless Propagation Letters, vol. 8, pp. $84-87,2009$.

[10] H. Kirino and K. Ogawa, "A $76 \mathrm{GHz}$ multi-layered phased array antenna using a non-metal contact metamaterial waveguide," IEEE Transactions on Antennas and Propagation, vol. 60, no. 2, pp. 840-853, 2012.

[11] E. Rajo-Iglesias and P.-S. Kildal, "Groove gap waveguide: A rectangular waveguide between contactless metal plates enabled by parallel-plate cut-off," in 4th European Conference on Antennas and Propagation. IEEE, 2010, pp. 1-4.

[12] A. J. Sáez, A. Valero-Nogueira, J. I. Herranz, and B. Bernardo, "Singlelayer cavity-backed slot array fed by groove gap waveguide," IEEE Antennas and Wireless Propagation Letters, vol. 15, pp. 1402-1405, 2015. 